\title{
Magnetic resonance urography in the assesment of congenital urinary system dilatation in pediatric patients
}

\author{
Rabia Mihriban Kılınça*, Irmak Durur Subası ${ }^{b}$, Afak Durur Kaya ${ }^{\mathrm{c}}$ \\ a Department of Radiology, Faculty of Medicine, Muğla Sitkı Kocman University, Muğla, Turkey \\ ${ }^{b}$ Department of Radiology, Faculty of Medicine, Atatürk University, Erzurum, Turkey \\ ${ }^{c}$ Department of Radiology, Erzurum State Research and Training Hospital, Erzurum, Turkey
}

\section{ARTICLE INFO}

\section{ABSTRACT}

\section{Article History \\ Received \\ 04 / 05 / 2013 \\ Accepted $\quad 21 / 05 / 2013$}

\section{* Correspondence to: \\ Rabia Mihriban Kılınç \\ Department of Radiology, \\ Faculty of Medicine, \\ Mugla Sitkı Kocman University, \\ Muğla, Turkey \\ e-mail: rabiaguner@hotmail.com}

\section{Keywords:}

Magnetic resonance functional imaging

Magnetic resonance urography

Pediatric patient

Urinary anomalies

\begin{abstract}
The aim of this study is to assess combined static-dynamic magnetic resonance (MR) urography in the evaluation of urinary tract anomalies in infants and children. Thirthynine patients with urinary tract anomalies underwent retrospective examination between 2009- 2012, with combined static-dynamic MR urography. A combination examination involved use of a static T2-weighted thick slap spin-echo sequence and a dynamic T1weighted tree-dimensional spoiled gradient-echo- recalled sequence with gadopentetate dimeglumine-DTPA and furosemide application. Morphologic results were compared with those of ultrasonography and, when available, surgery. The dynamic sequence was used to calculate renal transit time (RTT), split renal function from renograms generated from parenchymal regions of interest and to assess urinary excretion from whole-kidney renograms. Results were compared with those of diuretic renal scintigraphy for split function and urinary excretion. Urinary system dilatations caused by stenosis at the ureteropelvic $(n=16)$, ureterovesical $(n=4)$ junctions and within the ureter $(n=6)$, posterior urethral valve $(n=1)$, postoperative urethral obstruction $(n=1)$, neurogenic bladder $(n=1)$ and nonstenotic dilatation $(\mathrm{n}=4)$ and urinary tract anomalies, double collecting system $(n=4)$ and isolated urinary tract anomalies $(n=2)$ were clearly depicted. For morphologic evaluation, sensivity of MR urography compared with surgery calculated $82.75 \%$ and for split renal function, sensivity of dynamic MR urography compared with diuretic renal scintigraphy (DRS) calculated $80 \%$. For urinary excretion, MR urography and DRS showed strong agreement. MR urografi is a modality with which urinary system pathologies could be both morphologically and functionally evaluated with high accuracy and does not use ionizing radiation. But it has disadvantages for example, children need sedation during examination, results cannot be compared with enough clinical trials because of ethical reasons and some technical incapabilities. But we think with some technical improvements, MR urography can be applied in diagnosis and follow up of urinary system abnormalities in children with high degree of confidency.
\end{abstract}

J. Exp. Clin. Med., 2013; 30: 215-223

(C) 2013 OMU

\section{Introduction}

Imaging of urinary system on children has a fundamental value on the diagnosis of many congenital and acquired diseases. On the patients with congenital dilatation in urinary system, functional and anatomic information are required together on making decision for follow-up or operation. For this purpose, ultrasonography (US), diuretic renal scintigraphy (DRS), intravenous urography (IVU) and voiding cystourethrography have been used for years as conventional diagnostic methods (Rohrschneider et al., 2002).
Magnetic resonance (MR) imaging appears as an alternative method to the conventional examinations in the examination of the urinary system. MR urography provides sufficient information on the anatomy and physiology of urinary system. Nonuse of ionized radiation, the high contrast and spatial resolution of the images obtained on each three plane compose the most important advantages of MR urography. In the Heavy T2 weighted images, images are obtained by using the static liquid feature of the urine in the urinary system without giving contrast agent. The urinary system function 
after contrast agent can be assessed with T1 weighted (T1A) dynamic gradient echo sequences (Borthne et al., 2000; Riccabona et al., 2002; Rohrschneider et al., 2002). This study has been performed in order to assess and compare together the morphological-functional findings of MR urography, US and DRS on children with the diagnosis of congenital abnormalities and dilatation in upper urinary system by US and to determine the contributions of MR urography to the diagnosis and follow-up.

\section{Material and method Study Group}

The study has been performed through retrospective assessment on patients who applied to Erzurum State Research and Training Hospital Department of Urology and Pediatry and on who MR urography study was performed between 2009 and 2012 out of forty-two patients in ages between 1 month and 17 years, MR urography examinations were made on totally 39 patients composed of 25 boys and 14 girls within 3 years period. While 13 patients examined were younger than 1 year old, 13 patients were between 1-6 ages. Thirteen patients were between 6-17 ages. Three of the patients were also undergone postoperative control examination.

\section{Patients preparation}

During the examinations, the parents of the patients were routinely advised to allow their children sleep lesser before examination and to give breast milk or baby food and thus it was tried to form a sedative effect. These measures were sufficient for the children younger than six months $(n=6)$. In all of the children younger than 6 years and older than 6 months sedation was required $(n=20)$. For the children older than 6 years sedation was not required. These patients were examined after appropriate verbal information.

Intravenous (IV) line was opened in all patients before examination. In accordance with the protocol in our unit, serum physiologic bolus infusion of $10 \mathrm{ml} / \mathrm{kg}$ dose was started 30 minutes before the examination and it was maintained as $8 \mathrm{ml} / \mathrm{kg}$ /hour during the examination. The patients who have toilet training were sent to toilet for the bladder to be drained. On the patients for whom sedation was required, oral chloral hydrate in a dose of $50 \mathrm{mg} / \mathrm{kg}$ was used in accordance with the routine protocol. Again in accordance with the pediatric examination routine, the parents were taken to the examination room with the patient.

\section{Technics}

All of the MR urography examinations were performed by means of 1.5 -Tesla system (Magnetom, Symphony Siemens, Germany) device. The examinations were made in supine position. The examination was started by taking location determiner sections on three plans and then imaging was obtained in SSSE (single shot spin echo) sequences in coronal and axial plans to support the anatomic evaluation and to ensure the correct localization of the urinary system in other sequences. Immediately before taking axial sections (F-15 protocol) furosemide (Lasix ;Avetis Pharma, Bad Soden, Germany) in a dose of $0.3 \mathrm{mg} / \mathrm{kg}$ was applied as IV bolus. Under the guidance of locator images T1A and fat supressed T2A images including the kidneys, ureters and the whole of the bladder were obtained and anatomic imaging was completed with slab T2 images in coronal plan.

Contrasted T1A 3D spoiled gradient-echo-recalled (SPGR) sequence was used for dynamic examination (Table 1). The distance between the symphysis pubis and diaphragma was scanned in coronal plan slightly angled to include renal arteries in anterior, the whole of the kidney parenchyma in posterior and ureterovesical junctions. The examination periods were ranging between 12 and 15 seconds. The first repetition was taken without contrast and immediately then it was continued by taking sections with IV bolus contrast injection in a dose of $0.1 \mathrm{mmol} / \mathrm{kg}$. The procedure was repeated successively in first five minutes and per minute in the following five minutes in accordance with the routine dynamic examination protocol. The examinations were continued until whom the $20^{\text {th }}$ minute with five minute intervals on the patients in ureter contrasting was not observed within that period. The average examination period was 45 minutes.

Maximum intensity projection (MIP) and three dimension reconstructions were composed in a way to show the vascular structures and collecting system from the sections of the first repetition after the contrast agent was given.

For the purpose of functional evaluation, time-intensity curves were composed by manually selecting two different region of interest (ROI) in the contrasted sections from archive images. The first ROI was drawn on the kidney parenchyma as to include cortex and medulla (parenchymal ROI), the other was drawn as to include parenchyma and the pelvicaliceal system (whole kidney ROI). Thus, parenchymal renogram curves and whole kidney renograms showing the change of the average intensities in the selected area by time were composed. The parenchymal renogram curves were used to calculate the single kidney function and the whole kidney renogram curves were used to evaluate the excretory function.

\section{Assesment}

MR urography images were assessed by two radiologists and the results obtained by consensus were compared with urinary system ultrasonography examination for morphological assessment and with diuretic renal scintigraphy for functional assessment.

The dilatation in kidney collecting systems and urinary system features as well as pathologies such as stone, cyst and bulk were assessed by US before MR urography.

Morphological characteristics of the urinary system were first examined in SSSE, T1A, fat supressed T2A and slab heavy T2A sequences. Localization and size of the kidneys, parenchyma structure and thickness, caliceal structures, renal pelvis size, ureteropelvic junction, ureters, bladder and pararenal areas were assessed. The assessment of renal arteries and veins became possible with the images obtained from contrasted series. Furthermore the contrasted images enabled the detailed assessment of the contrasting pattern of two kidney parenchyma, the accumulation and homogeneity of contrast agent in pelvicaliceal structures, ureteropelvic junctions, structures of the ureters, calibration, ureterovesical junction and structural characteristics of the bladder.

Parenchymal renogram curves were used for functional assessment. The renogram curves of the normal kidney on the patients with unilateral pathology were compared with the other in these curves. Maximum intensity values of the 

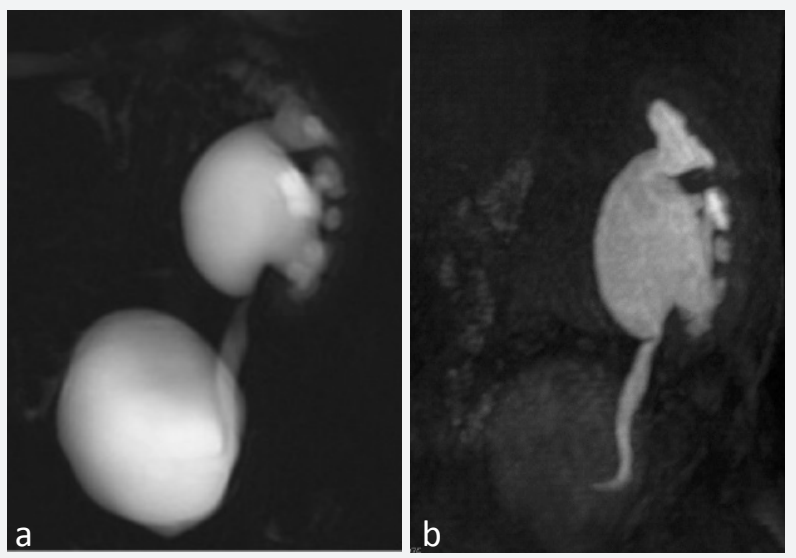

Fig. 1. Two years old male patient. IV grade dilatation in left kidney is being monitored in thick slab heavy $\mathrm{T} 2$ (a) and post contrast $20^{\text {th }}$ minute T1A MIP (b) images. Right kidney cannot be monitored in the patient diagnosed with left UPJO (agenesia).

parenchyma were compared. Also single kidney functions were calculated on some patients.

Urinary excretion was assessed from whole kidney renogram curves and by renal transit time (RTT) calculation. According to this; if accumulation curve composed in whole kidney renogram and the visual irrigation from renal pelvis is minimal or delayed, obstruction was diagnosed in that kidney. If contrast agent was not seen in collecting system in kidneys, this situation was considered as in favor of significant decrease in concentration capacity and accepted as low functional- nonfunctional kidney. Moreover according to RTT; the grades were normal for below 4 minutes, suspicious for 4-8 minutes, obstruction for over 8 minutes and serious obstruction for over 15 minutes.

The excretory function of the urinary system was included in the study together with the single kidney function in the diuretic renal scintigraphy. According to this the meaning of the grades are; 0: Normal, 1: Extension of the excretion and partial obstruction, 2: Insufficient response to diuretic and significant obstruction.

\section{Findings}

In our study, $42 \mathrm{MR}$ urography examinations were performed on 39 patients whose pathologies were detected in urinary system via US. Three of the patients were undergone MR urography examination before and after operation. Routine protocol was used on all patients (Table 1). It was learned that the examination could not be completed as the sedation of 10 patients who are referred after the diagnosis of urinary system pathology could not be achieved. Sedation was performed on
20 of the patients. Diuretic renal scintigraphy examinations of 30 patients were performed in our hospital or in another center.

\section{Morphological findings}

Ureteropelvic junction obstruction (UPJO) was diagnosed in 16 of 39 patients in our study. Four of them were bilateral, 11 left and 1 right UPJO. Also one of them was assessed after bilateral pyeloplasty operation. On 3 of the patients both preoperative and postoperative MR urography examinations were performed.

On all of the patients with UPJO, dilatation on renal pelvis, blunting on calyxes, decrease in the thickness of parenchyma, ballooning of renal pelvis, pelvis structure narrowing by excelling in ureteropelvic junction was defined in various levels (Fig. 1). It was observed on the sections taken after contrast that contrast agent shows leveling in dilated pelvicaliceal system.
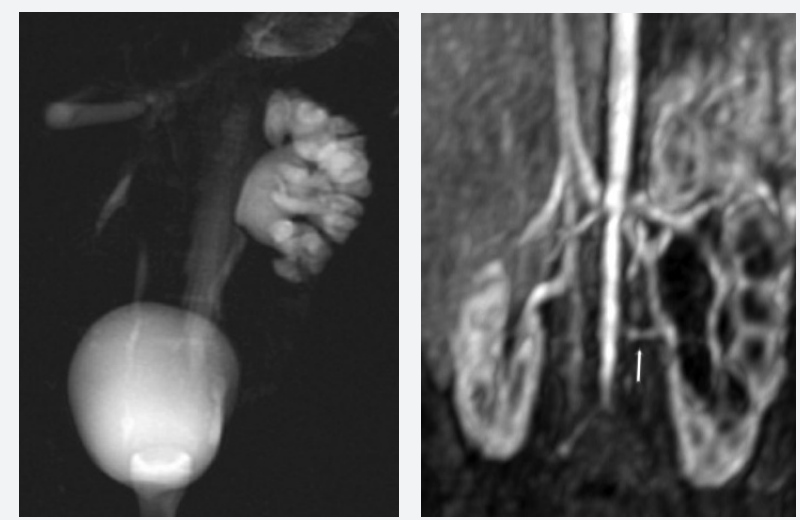

Fig. 2. Six months old female patient. Dilatation on renal pelvis in left kidney, blunting on calyxes, ballooning of renal pelvis, pelvis structure narrowing by excelling in ureteropelvic junction is monitored in heavy $\mathrm{T} 2 \mathrm{imag}$ es in patient diagnosed with left UPJO. Also accessory renal artery intercrossing renal pelvis is seen in post contrast series in arterial phase (arrow).

One of the patients diagnosed with unilateral UPJO, contralateral kidney could not be diagnosed in US, MR urography and scintigraphy. The patient had no operation history. Thus that patient was evaluated as renal agenesis. In a patient with left UPJO, calyx calculus was seen in right kidney in US. But the calculus could not be seen in MR urography.

In three of the patients diagnosed as unilateral severe UPJO, accessory renal artery intercrossing renal pelvis was seen in post contrast arterial examination (Fig. 2). In one of these patients renal artery intercrossing pelvis was not described in US.

Table 1. Parameters of MR sequences

\begin{tabular}{|c|c|c|c|c|c|c|c|c|}
\hline Sequences & $\begin{array}{c}\text { TR } \\
(\mathbf{m s n})\end{array}$ & TI & $\begin{array}{c}\text { TE } \\
(\mathbf{m s n})\end{array}$ & $\begin{array}{l}\text { FA } \\
(d g)\end{array}$ & $\begin{array}{c}\text { KK/gap } \\
(\mathrm{mm})\end{array}$ & FOV & $\begin{array}{c}\text { Matrix } \\
(\mathrm{mm})\end{array}$ & nex \\
\hline Pilot T1A GRE & 15 & - & 5 & 40 & $10.0 / 0.2$ & & $256 \times 256$ & \\
\hline SSSE(ax and cor) & 2500 & - & 60 & 90 & $8.0 / 0.0$ & $30 \times 20$ & $256 \times 256$ & 0.54 \\
\hline T1A(ax) & 700 & - & 20 & 90 & $5.0 / 1.0$ & $30 \times 30$ & $288 \times 224$ & 2.0 \\
\hline $\mathbf{T} 2 \mathrm{~A}(\mathbf{a x})$ & 4000 & - & 105 & 90 & $5.0 / 1.0$ & $30 \times 22$ & $288 \times 224$ & 2.0 \\
\hline Thick 'slab' heavyly T2 & 5150 & - & 1030 & 90 & $40.0 / 0.0$ & $28 \times 28$ & $256 \times 256$ & 0.94 \\
\hline T1A 3D GRE with contrast (cor) & 5.3 & 21 & 1.7 & 30 & $2.0 /-1.0$ & $48 \times 24$ & $256 \times 160$ & 0.5 \\
\hline
\end{tabular}

GRE: Gradient echo, SSSE: Single shot spin echo, ax: Axial, cor: coronal, msec: milisecond, dg: degree, st: Section thickness, mm: Millimeter, nex: Number of excitation. 

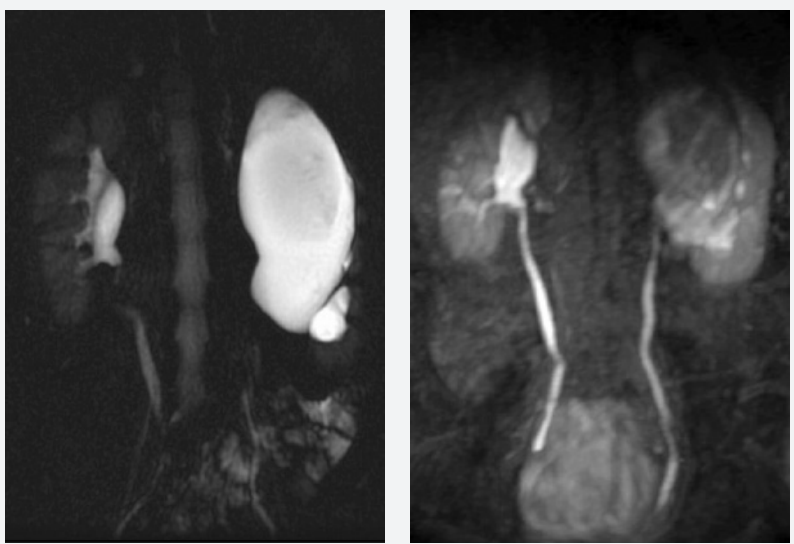

Fig. 3. Seventeen years old male patient. Liquid content structure that does not show significant contrasting in the upper side of the left kidney of the patient is seen in heavy $\mathrm{T} 2$ and post contrast $10^{\text {th }}$ minute post contrast images. The patient who was diagnosed with parapelvic cyst in ultrasonography and MR urography was diagnosed UPJO during the operation.

On four patients diagnosed as unilateral UPJO postoperative MR urography control was performed. Besides the diagnosis of morphological UPJO findings, healing findings such as decrease in the size of renal pelvis, increase in the parenchymal thickness and significant acceleration in the pass of contrast to the ureter were determined in these patients.

MR urography was found successful on diagnosing pathology in all patients except one. On this patient who was diagnosed as left UPJO during operation, widened pelvis suppressed the parenchyma on the upper side of the kidney and severely slimmed it. The lower side was normal. So the patient was evaluated as parapelvic cyst or calyx diverticulum (Fig. 3).

The other group was composed of patients diagnosed as hydroureteronephrosis (HUN) in the pathologies examined. There were 12 patients four of whom were bilateral. In the patients diagnosed with HUN in MR urography, collecting system dilatation continuing to the ureter distal and parenchymal slimming in various levels and ureteric tortuosity was observed.

Width of ureterovesical junctions was an important criterion in determining the etiology of HUN. Also intraluminal pathologies were also tried to be assessed. In 5 of the patients diagnosed with HUN in the ultrasonography, distal ureter calculus was defined in the etiology of the dilatation. Only one of these patients could be diagnosed by MR urography. In three of 5 patients, ipsilateral or contralateral calculus were determined by US and could not be observed in MR urography.

In MR urography of four patients diagnosed with unilateral HUN, ureterovesical junctions were apparently narrow in spite of the dilatation of collecting system. These patients were evaluated as ureterovesical junction obstruction (UVJO). The operation results of these patients that were evaluated as UPVO supported the diagnosis.

In a patient operated before due to posterior urethral valve, bilateral severe HUN was diagnosed again by US. In MR urography, severe HUN and dilatation in ureterovesical junctions were seen in this patient. Contrast pass did not hap- pen to the right kidney collecting system in $20^{\text {th }}$ minute and it was diagnosed as right dysfunctional kidney. Also RTT was longer than 20 minutes for the left kidney. This situation was evaluated as in favor of severe obstruction. Postoperative cicatricial obstruction in urethra presence was thought in the patient and the patient was evaluated as tertiary HUN.

Severe HUN accompanied by severe slimming in bilateral parenchyma was observed in a patient. Ureterovesical junctions were also apparently dilated. The renal transit time of the patient was over 20 minutes for each kidney. Thus obstructive pathologies were evaluated. MR result of the patient was coherent with posterior urethral valve and the diagnosis was verified by the operation.

In a patient followed-up due to neurogenic bladder severe left HUN was diagnosed. Pathology was not observed in ureterovesical junction and RTT was calculated as six minutes. Severe obstruction was not evaluated for the patient. The patient on whom grade V reflux on left was observed by voiding cystourography (VSUG), was diagnosed with neurogenic bladder and reflux.

The last group of the patients in our study had double collecting system. The patients were defined as severe HUN
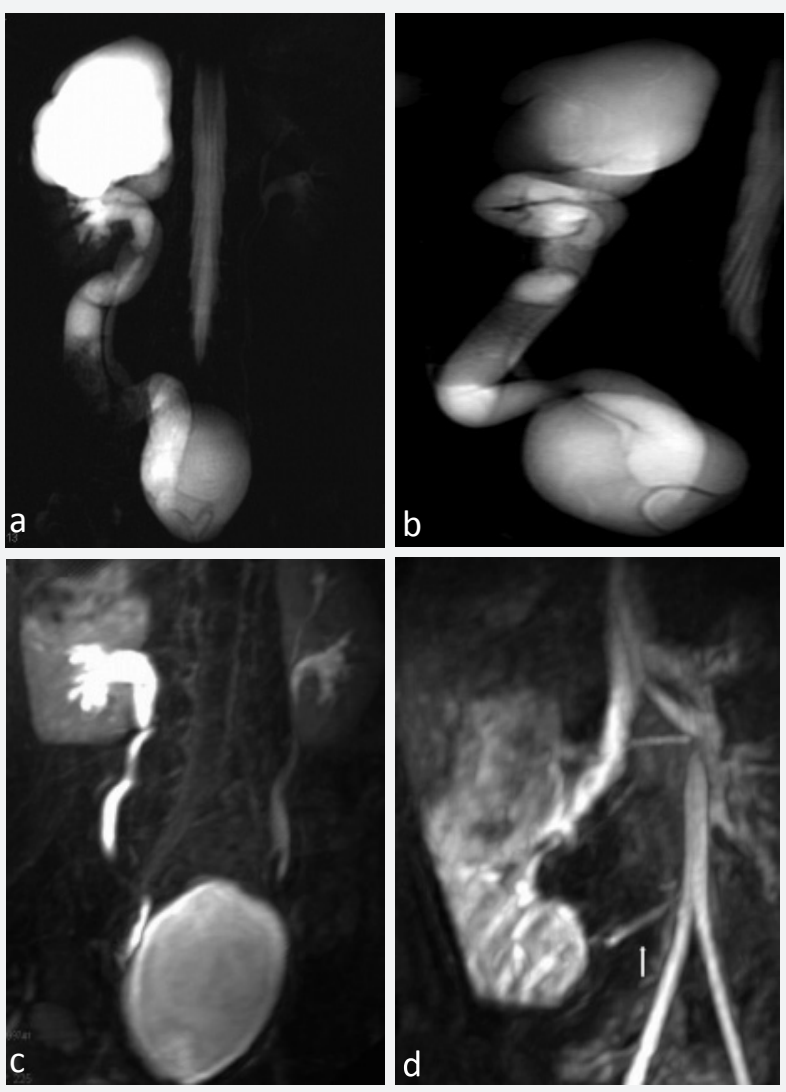

Fig. 4. Seven years old male patient. Bilateral double collecting system abnormality is seen in heavy T2 images $(\mathbf{a}, \mathbf{b})$. The upper system collecting system in right kidney is dilated and ureter is tortuous to the distal. Right upper system ureter presents ectopic opening from the inferior of the lower system to the bladder (B, long arrows). Contrast agent is not observed in the collecting system of right upper system at the end of the $20^{\text {th }}$ minute in post contrast series. Apparent mild dilatation is present in pelvis in the right lower system (c). Accessory renal artery intercrossing the pelvis of right lower system was seen in post contrast arterial phase (arrow). 

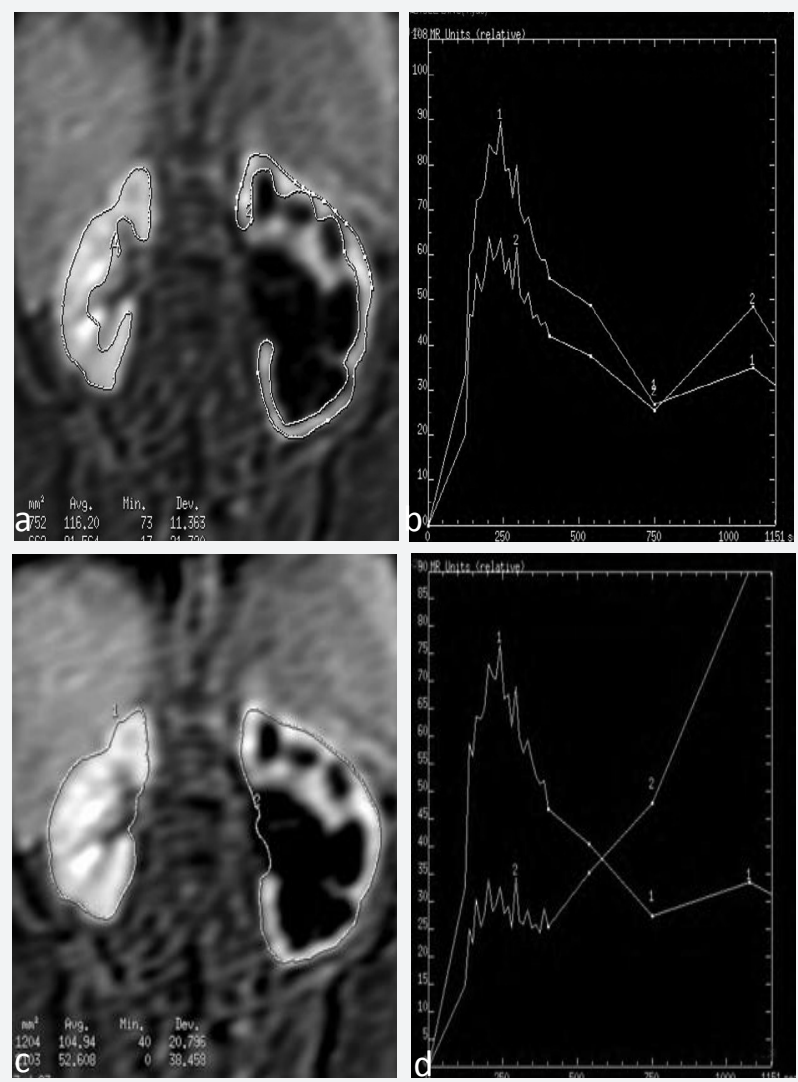

Fig. 5. Two years old male patient. Parenchymal (a, b), complete kidney (c, d) renogram curves of the patient diagnosed with left UPJO. It is seen in the parenchymal renogram curve that maximum parenchyma intensity of right kidney (1) is significantly higher than of the left. The single kidney function of this patient was found as L 30\%, R $70 \%$ by MR urography and as L $36 \%$, R $64 \%$ by renal scintigraphy. In complete kidney curves, the 3rd segment starting from the maximum point in right kidney shows a rapid decrease but composes accumulation curve on left by constantly increasing. Left kidney RTZ of this patient was over 15 minutes.

in double collecting system and upper systems in the ultrasonography. One patient was reported as ectopic urethra opening and two patients were reported as ectopic bladder opening (Fig. 4). The distal ureter opening of a patient could not be seen due to gas artifacts. Complete or partial double collecting systems evaluation was not made for the kidney, which did not show dilatation, of the patient with bilateral double collecting system. Complete double collecting system was observed in magnetic resonance urographies in all of the patients (totally 5 ureter-kidney units) and severe HUN was monitored in unilateral upper systems. In two of the patients ectopic opening dilated upper system ureters to the upper side of the vagina and in two of them to the bladder. Accessory renal artery intercrossing dilated pelvis was found in post contrast series. RTT was calculated as 5 minutes for lower system in this patient and evaluated as moderate UPJO in right lower system. In this patient and in 2 patients with ectopic ureter opening to vagina, contrast pass to the collecting system was not seen in upper system (dysfunctional upper systems). In the other patient with ectopic opening to bladder, the renal transit time was 16 minutes. Moderate UVBO was evaluated on this patient.
Isolated urinary system abnormalities were present in 4 patients out of the mentioned patients. In a patient in which grade 3 hydronephrosis (HN) in left and dilatation in ureter proximal was diagnosed in ultrasonography, dilatation was seen in left kidney pelvicaliceal system and the first $4 \mathrm{~cm}$ section of ureter in MR urography. Dilatation was ending sharply in the ureter. Renal transit time was over 8 minutes that indicates obstruction (10 minutes). Incomplete valve at level of obstruction was described in ureter in post contrast series. The patient was operated due to left ureteric "web" and folding and obstruction was observed in that area.

Soft tissue mass covering left renal artery in paraaortic area was found in MR urography performed up on the observation of atrophy in left kidney by US on a patient followed up due to operated neuroblastoma. Size of the lesion was compared with the abdominopelvic tomographies performed previously and no significant increase in size was seen, the findings were evaluated as secondary soft tissue changes and obstruction in left renal artery to operation and/or radiotherapy.

Twenty-nine of 39 patients examined were operated due to urinary system pathologies. Ten patients were started to be
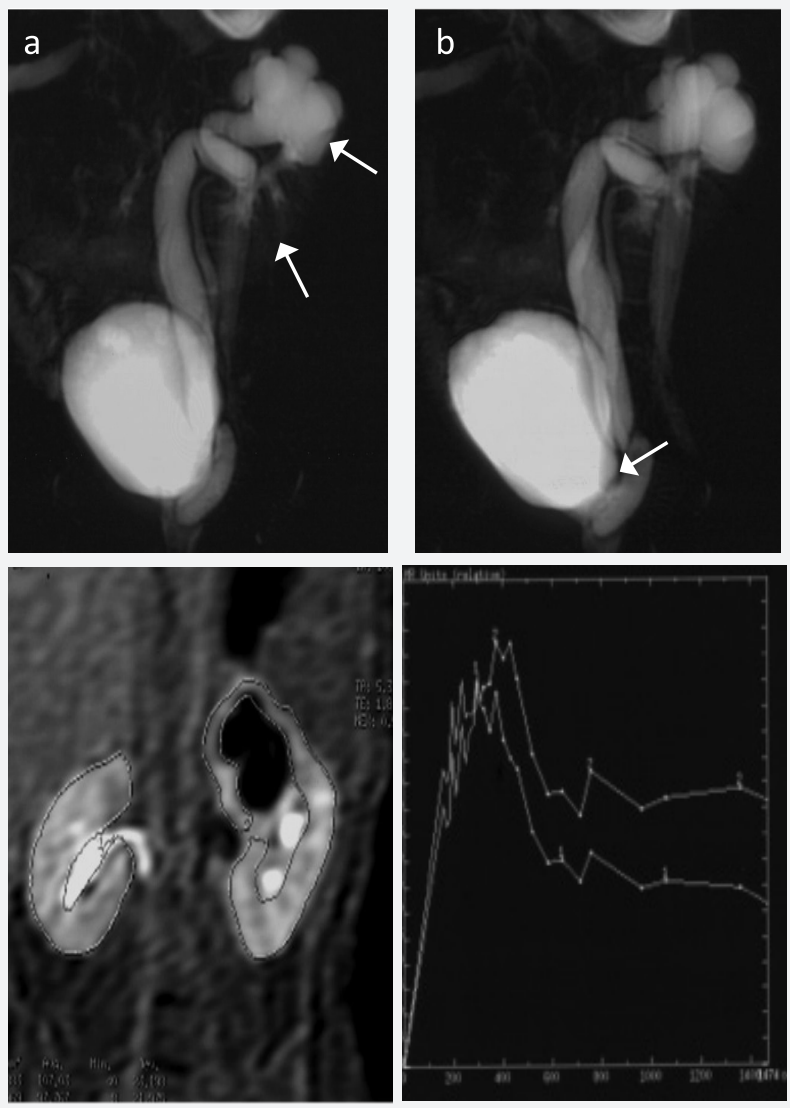

Fig. 6. One year old female patient. Left kidney with double collecting system (long arrows) on which more apparent dilatation is observed is monitored in the upper side on left in heavy T2 images (a). Left upper system ureter was ending on the upper side of the vagina presenting tortuosity and dilatation up to the distal $(\mathbf{b}$, arrow). When the parenchymal renogram curves of the patient were compared it was seen that the curves were similar. While the single kidney functions (including the complete parenchyma of the left kidney) of this patient was found as L $45 \%$, R 55\% by MR urography, it was found as $\mathrm{L} 48 \%, \mathrm{R}$ $52 \%$ in diuretic renal scintigraphy. 

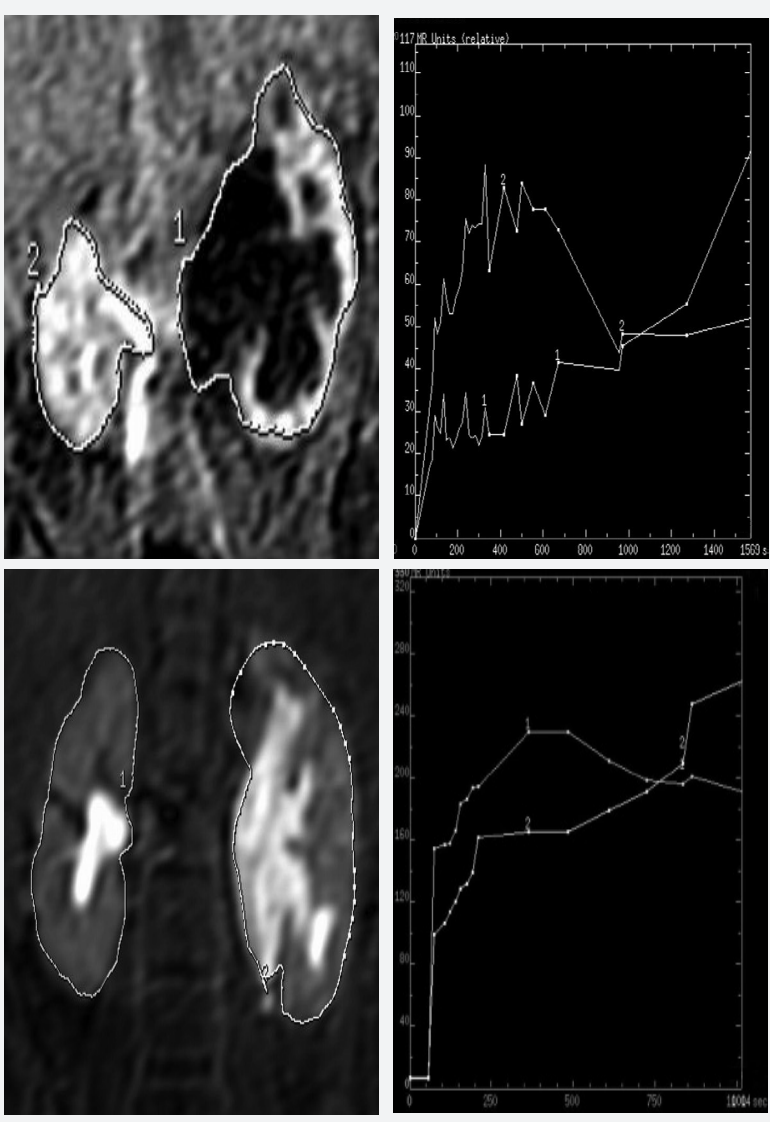

Fig. 7. One month old female patient. In preoperative (upper line) and post operative (lower line) complete renogram curves, it is observed that apparent accumulation curve forms before operation. Accumulation curve continues after the operation. While the RTZ of the patients is preoperative over 20 minutes, they are 5 minutes in postoperative examination. When evaluated with morphological findings it was thought that the obstruction in the left kidney regressed. The formation of accumulation curve in the postoperative period can be correlated with the continuation of some obstruction or the amplification effect of MR urographic complete kidney renograms on accumulation.

followed. Morphological findings obtained by MR urography were compared with operation findings in patients operated and with US findings in all patients. Urinary system dilatation in all patients was observed as coherent with US and in similar grades. But MR urography was unsuccessful to determine the cause of the dilatation in 5 of the patients operated. One of these patients were diagnosed UPJO and four of them were nephro-ureterolithiasis. Urinary system calculus was seen by ultrasonography, calyx diverticulum -cyst definition was done in patient with UPJO similarly in the MR urography. According to this, the sensitivity of MR urography in determining the etiology of dilatation was calculated as $82.75 \%$ when compared with operation findings.

\section{Functional Findings}

There were 20 patients both of whose diuretic renal scintigraphy and MR urographic parenchymal renogram curves could be obtained. Four of them had single kidneys and could not be undergone comparative evaluation. Thus single kidney functional values were compared in totally 16 patients.
The calculated function of pathological kidney was compared with the value in the scintigraphic examination (Table 2).

MR urographic parenchymal renograms of all patients were obtained. The maximum intensity value in these renograms were compared with each other for right and left kidneys. The maximum intensity of the pathological kidneys of 7 patients was found as lower than their symmetry according to this (Fig. 5). The curves were similar for both two kidneys in nine patients and it was evaluated in favor that no apparent functional loss was present in the pathologic kidney (Fig. 6). Bilateral urinary system dilatation was found in three patients. In the renograms of these patients it drew attention that both of the 2 kidneys composed curves in similar patterns. But their maximum intensities were significantly lower in comparison to the other patients. Thus it was thought that the total functions of the kidneys decreased.

The sensitivity and specifity of MR urography in functional calculation was calculated as $80 \%$ when compared with DRS according to these results.

\section{Assessment findings of urinary excretion}

Complete kidney renogram curves on all of 39 patients in our study were obtained. Accumulation curves of these patients were observed in a way that could indicate obstruction in all described as 3-4 grade dilatation by US $(n=31)$. However RTT was not at an obstructive level in three of these patients. Two of them were followed due to bilateral HUN, the other one was diagnosed unilateral ureterolithiasis. 30 patients whose renal transit time was at obstructive level were determined.

Twenty-nine of all patients were operated. Obstruction in various levels was observed in twenty six patients. Dysfunctional double collecting system abnormality was present in three patients and partial nephrectomy was applied on these patients. RTT was found as high in 23 of these 26 patients. According to this, renal transit time and obstruction presence could be found with $88 \%$ sensitivity in MR urography.

Accumulation curve appearance was constant in the renograms of 4 patients examined due to ureteropelvic junction

\begin{tabular}{|c|c|c|c|c|c|}
\hline & \multirow[t]{2}{*}{$\begin{array}{c}\text { Patient } \\
\text { name }\end{array}$} & \multirow{2}{*}{$\begin{array}{l}\text { DRS } \\
\text { L (\%) }\end{array}$} & \multicolumn{3}{|c|}{$\begin{array}{c}\text { MR } \\
\text { urography }\end{array}$} \\
\hline & & & $\mathrm{R}(\%)$ & $\mathrm{L}(\%)$ & $\mathrm{R}(\%)$ \\
\hline 1 & $\mathrm{AU}$ & 50 & 50 & 49 & 51 \\
\hline 2 & BMK & 61 & 39 & 46 & 54 \\
\hline 3 & MRK* & 44 & 56 & 48 & 52 \\
\hline 4 & $\mathrm{BF}$ & 24 & 76 & 24 & 76 \\
\hline 5 & UE & 52 & 48 & 54 & 46 \\
\hline 6 & GÇ & 45 & 55 & 34 & 65 \\
\hline 7 & $\mathrm{CC}$ & 50 & 50 & 51 & 49 \\
\hline 8 & YK & 55 & 45 & 59 & 41 \\
\hline 9 & GEK & 67 & 33 & 40 & 60 \\
\hline 10 & $\mathrm{ADD}$ & 48 & 52 & 40 & 60 \\
\hline 11 & ASA & 36 & 64 & 30 & 70 \\
\hline 12 & HR & 36 & 64 & 25 & 75 \\
\hline 13 & $\dot{\mathrm{IIK}}$ & 48 & 52 & 47 & 53 \\
\hline 14 & ENK & 50 & 50 & 51 & 49 \\
\hline 15 & İK & 55 & 45 & 52 & 49 \\
\hline 16 & MB & - & 100 & 2 & 98 \\
\hline
\end{tabular}


obstruction, obtained after operation. But their RTTs shortened. It was concluded that the obstruction in the patients significantly regressed through this information (Fig. 7).

\section{Discussion}

Ultrasonography has been the basic examination used in the monitoring of the urinary system pathologies of the patients in pediatric group and in diagnosis and follow-up for years. Moreover VSUG in the diagnosis of vesicouretheral reflux (VUR) and diuretic real scintigraphy in the evaluation of renal functions are the monitoring methods applied. MR urography is a monitoring method relatively new in comparison to others and a preferred one as it presents accurate anatomic information and functional data, does not include ionized radiation and for the wide safety range of the contrast agent used and the obtainment of quick images (Borthne et al., 2000; Hwang et al., 2000; Rohrschneider et al., 2000a, Rohrschneider et al., 2000b; Zielonko et al., 2003). In this study, the morphological and functional diagnostic characteristics of MR urography, US and diuretic renal scintigraphy were discussed on the pediatric patients which were diagnosed with complex urinary system abnormalities and collecting system dilatation by US in our clinic.

Safe sedation of pediatric patients has an importance in terms of examination quality in radiology. The sedative effect of breast feeding can be benefited for babies. Making the patients sleep lesser before examination also contributes to sedation (Frush et al., 1996). Sedation was applied on 20 patients between 6 months and 6 ages with oral chloral hydrate in a dose of $50 \mathrm{mg} / \mathrm{kg}$.

Application of low dose diuretic before gadolinium enables the complete and uniform contrast increase (Semelka et al., 1990; Rohrschneider et al., 2000a, Rohrschneider et al., 2000b; Verswijvel et al., 2000; Nolte-Ernsting et al., 2001). In the studies performed by comparing gradient echo sequences in which only gadolinium is used without diuretic, it was asserted that anatomic details are monitored better in the combination of diuretic and gadolinium. Diuretic in a dose of $0.1 \mathrm{mg} / \mathrm{kg}$ and gadolinium in a dose of $0.1 \mathrm{mmol} / \mathrm{kg}$ was given to all of 39 patients in our study.

Monitoring of collecting system and ureters by IVU can last very long and cannot be possible on some patients, in the systems in which the kidney functions decrease and which are severely dilated (Prasad and Priatna, 1999; Riccabona et al., 2002; Zielonko et al., 2003). The same patients were evaluated by MR urography and pathology was diagnosed in the first 13 seconds in coronal heavy T2 sequence. In our study axial fat surrounding T2A sequence was also taken together with thick 'slab' heavy T2 sequence. Also the anatomic monitoring was supported with T1A axial and (to relatively avoid from movement artifacts) SSSE images. Urinary systems could not be monitored in patients with severe urinary system dilatation in post contrast sections similarly to IVU and in 1 patient. Dilated urinary system was easily monitored by heavy T2 images in these patients. Axial sections enabled the assessment of dysfunctional kidney.

Riccabona et al. (2002) made a study on 29 newborns and babies with upper urinary system abnormality. Three dimensional GRE sequences were taken to the patients diagnosed with abnormality in US after coronal HASTE and contrast agent. Ureteropelvic junction obstruction, megaureter, VUR, double collecting system and renal parenchymal disease diagnoses were compared with the results of US, IVU, VSUG and scintigraphy. It was reported that MR urography has a better image quality and is better than IVU in anatomic assessment. It was found out that MR uroghraphy does not have superiority against US in determining renal parenchyma and obstruction. Kidneys with severe obstruction and low function could not be monitored in IVU. MR urography provided more accurate information than US and IVU in severe obstructions, genital malformations, ectopic kidney and ureter insertion. MR urography was found superior than US in showing not dilated systems and ureteral pathologies. Hvvang et al. (2000) compared the efficiency of HASTE MR urography and US on 12 patients on which urinary system could not be opacified sufficiently with IVU. MR urography was found as more efficient than US in determining the level and cause of the obstruction (Gaeta et al., 1999). MR urography and US were found as similar in diagnosing renal parenchyma structure and obstruction. However US was more successful than MR urography in monitoring the pathology causing dilatation on patients with urinary system dilatation, especially in detecting calculus. Ureters could be assessed as more detailed, as the morphological characteristics of the urinary system could be monitored completely by magnetic resonance urography. MR urography was better than US in monitoring the course of ureters in dilated and non dilated systems and determining the insertion area as well as differentiating partial and complete double collecting systems especially on patients with double collecting systems.

The results achieved in the studies performed to evaluate MR urography and renal function numerically in literature are towards the possibility that it can be the alternative of the diuretic renal scintigraphy (Borthne et al., 2000; Avni et al., 2002; Rohrschneider et al., 2002; Zielonko et al., 2003). A dynamic MR urography technique that enables the calculation of single kidney function similarly with the scintigraphy was used in our study. When the single kidney functions calculated by dynamic MR urography were compared with the values in renal scintigraphy, the sensitivity and specifity of MR urography was calculated as $80 \%$ and the intraclass correlation coefficient (ICC) was calculated as 0.90 ( $\mathrm{p}<0.001)$. This findings is coherent with the literature.

The assessment of urinary excretion in MR urography is made depending on the decrease of the $3^{\text {rd }}$ segment in complete kidney renograms similarly in scintigraphy. In normal kidneys, the $3^{\text {rd }}$ segment of the renogram presents a concave decrease in the way to show the efficiency of excretion of the agent to the collecting system. This decrease starts right after the maximum point (Sadowski et al., 2007). The curve typically turns back to the value accepted as the starting point ( $\mathrm{P}$ point) or the slightly lower value. This return delays or does not happen in obstruction.

Rohrschneider et al. (2002) reported that this normal pattern is seen in morphologically normal kidney-ureter units approved to show good irrigation with diuretic renal scintigraphy but the coherence of diuretic renal scitigraphy and MR urography in the diagnosis of urinary excretion in abnormal kidney-ureter units was approx. $80 \%$. It is stated that in the remaining side MR urography exaggerates excretion disorder. This situation can be derived from the use of different agents in the diuretic renal scintigraphy and 
dynamic MR urography. While gadolinium dimeglumineDTPA is primarily being emitted to glomerules from kidneys by filtration, Tc-99m MAG-3 is emitted by tubular secretion. This difference is not effective in the calculation of single kidney function in the chronic urinary system obstructions. But in the evaluation of urinary excretion, differences deriving from the more effective elimination of Tc-99m MAG-3 by the kidneys can occur. The decrease of the 3rd segment in the renograms in the collecting systems with dilatation may be slower with gadopentate dimeglumine-DTPA than with Tc99m MAG-3. Accumulation curve occurred in 30 patients in our study but RTT was not a obstructive level in 3 of them in spite of the curve formation. When the operation results of the patients anticipated as obstruction by renal transit time were compared, the sensitivity of MR urography was calculated as $88 \%$. Therefore it was thought that the calculation of RTT together with the complete kidney renogram curves in the assessment of urinary excretion can increase the sensitivity of MR urography in terms of the determination of obstruction presence.

MR urography does not include ionized radiation. This advantage gains important especially when repetitive examinations are needed on children and infants. Ureteropelvic and ureterovesical junction obstructions can improve with maturation (Cartwright et al., 1992; Korf and Campbell, 1992). On these types of patients with stenosis, control examinations should be done for the evaluation of kidney function and morphology with the clinical follow-up until the development of maturation or operation. US and scintigraphy controls are made annually or at more frequent intervals on these. The younger the patient the closer followup is required. In this respect a method that can substitute for scintigraphy and ultrasonography can be very beneficial. Also the static-dynamic MR urography enables the assessment of whole urinary system. Besides this, information about the kidney function and urinary excretion can be obtained.

In spite of these advantages of MR urography, morphological evaluation lacks a standard. All of the patients were not undergone surgery as in our study and some of them were followed-up with conservative treatment by conserving function. Even though the studies (Nolte-Ernsting et al., 2001; Zielonko et al., 2003) show that MR urography is superior to excretory urography in terms of morphological evaluation, these studies are not supported by clinical studies. Thus MR urographic findings are compared with US. But US has limitations in monitoring the whole urinary system.

One of the biggest disadvantages of MR urography is the need of sedation on patients that do not cooperate. Movement artifacts can happen as the sedative agents do not cause deep sedation and the agents causing deep sedation requires a separate anesthesia team and also they carry risks for the patients.

It was reported that gadolinium chelatagents have very low nephrotoxicity in clinical doses (Rohrschneider et al., 2002; Zielonko et al., 2003). However, articles stating that MRG or MRA examination with contrast agent containing gadolinium on patients with moderate kidney failure (glomerular filtration rate $<60 \mathrm{ml} / \mathrm{min}$ ) and severe kidney failure (glomerular filtration rate $<15 \mathrm{ml} / \mathrm{min}$ ) can cause a disease which is called Nephrogenic Systemic Fibrosis (NSF) and may be fatal, have been published in recent years (Sadowski et al., 2007). It is clear that evaluating the kidney function coherence of the patients to be examined by MR urography before the use of contrast and taking the necessary measures is compulsory.

\section{REFERENCES}

Avni, F.E., Bali, M.A., Regnault, M., 2002. MR urography in children. Eur. J. Radiol. 43, 154-166.

Borthne, A., Pierre-Jerome, C., Nordshus, Reiseter, T., 2000. MR urography in children: Current status and ruture development. Eur. Radiol. 10, 503-511.

Cartwright, P.C., Duckett, J.W., Keating, M.A., 1992. Managing apparent ureteropelvic junction obstruction in the newborn. J. Urol. 148, 12241228.

Frush, D.P, Bisset GS 3rd, Hail, S.C., 1996. Pediatric sedation in radiology: The practise of safe sleep. AJR. 167, 1381-1387.

Gaeta, M., Blandino, A., Scribano, E., 1999. Diagnostic pitfalls of breath-hold MR urography in obstructive uropathy. J. Comput. Assist. Tomogr. 23, 891-897.

Hwang, S.I., Kim SH, Kim YJ., 2000. Effectiveness of MR urography in the evaluation of kidney which failed to opacify during excretory urography: Comparison with ultrasonography. Korean J Radiol. 1, 152-158.

Korf, S.A., Campbell, K.D., 1992. Nonoperave management of unilateral neonatal hydronephrosis. J. Urol. 148, 525-531.

Nolte-Ernsting, C.C.A., Adam, G.B., Günther, R.W., 2001. MR urography. Examination techniques and clinical applications. Eur. Radiol. 11, 355-372.

Prasad, V.P., Priatna, A., 1999. Fuctional imaging of the kidneys with fast MRI tecniques. Eur. J. Radiol. 29, 133-148.

Riccabona, M., Simbninner, J., Ring, E., Ruppert-Kohlmayr, A., Ebner, F., Fotter, R., 2002. Feasibility of MR urograpy in neonates and infants with anomalies of the upper urinary tract. Eur. Radiol. 12, 1442-1450.

Rohrschneider, W.K., Hoffend, J., Becker, K., Clorius JH., Darge K., Kooijman H., Tröger J., 2000a. Combined static- dynamic MR urography for the simultaneous evaluation of morphology and function in urinary tract obstruction. Evaluation of the normal status in an animal model. Pediatr. Radiol. 30, 511-522.

Rohrschneider, W.K., Becker, K., Hoffend, J., 2000b. Combined static- dynamic MR urography for the simultaneous evaluation of morphology and function in urinary tract obstruction. Findings in experimentally induced üreteric stenosis. Pediatr. Radiol. 30, 523-532.

Rohrschneider, W.K., Haufe, S., Weisel, M., 2002. Functional and morphologic evaluation of congenital urinary tract dilatation by using combined static- dynamic MR urography: Findings in kidneys with a single collecting system. Radiology. 224, 683-694.

Sadowski, E.A., Bennett, L.K., Chan, M.R., Wentland, A.L., Garrett, A.L., Garrett, R.W., Djamali A., 2007. Nephrogenic systemic fibrosis: Risk factors and incidence estimation. Radiology. 243, 148-157.

Semelka, R.C., Hricak, H., Tomei, E., Floth A, Stoller M., 1990. Obstructive nephropathy: Evaluation with dynamic Gd- DTPA enhanced MR imaging. Radiology. 175, 797-803. 
Verswijvel, G.A., Oyen, R.H., Van Hoppel, H.P., 2000. Magnetic resonance imaging in the assessment of urologic disease: An all-in-one approach. Eur. Radiol. 10, 1614-1619.

Zielonko, J., Studniarek, M., Markuszevski, M., 2003. MR urography of obstructive uropathy: Diagnostic value of method in selected clinical groups. Eur. Radiol. 13, 802-809. 